\title{
Assessing the impact on less-than-full-time core medical trainees of a WhatsApp group and significantly enhanced website information
}

\author{
Authors: Sara Page ${ }^{A}$ and Matthew Roycroft ${ }^{B}$
}

\begin{abstract}
Aims
To see whether a combination of targeted changes improved the experience of core medical trainees (CMTs) in less-than-full-time training (LTFTT) in Yorkshire and the Humber (Y\&H). The changes introduced were: a new WhatsApp group for LTFT CMTs, and regional website updates and document creation targeted at LTFT trainees.
\end{abstract}

\section{Methods}

All CMTs working LTFT in Y\&H were invited to complete an online survey regarding LTFTT, from which two main themes were identified for improvement work: the desire for increased support and better information provision. To address support, a WhatsApp group open to all CMTs either working or considering working LTFT was created, and included a Health Education England (HEE) leadership fellow with good understanding of the LTFT system to respond to technical questions. To address information provision, new LTFT, parental leave and returning to work sections were created on the regional CMT website, providing key information, trainee stories, links to policies, and plain English frequently asked questions to go alongside the regional LTFT and return to training policies, but targeted specifically at medical trainees.

Six months after the initial survey and with the WhatsApp group and website changes established for many months, LTFT trainees were again asked to complete the same survey to assess progress. The survey questions were either in simple binary (yes/no) form or 5 -point scales.

\section{Results}

LTFTT is relatively infrequent in CMT (only $4 \%$ of trainees within our region). Out of the eight trainees presently working LTFT, six completed the initial survey and four the subsequent one. There is probably a degree of overlap in respondents between the two groups.

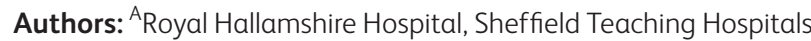

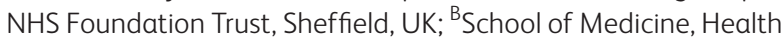
Education England, Yorkshire and the Humber, UK
As of January 2018, the WhatsApp group has 11 members and has active use, with an average of around 25 messages sent per month.

The percentage of trainees reporting knowing who to contact for support about LTFTT increased from $17 \%$ to $100 \%$.

Although some improvement was seen in how trainees viewed our website with regards to information on both maternity leave and return to work, it barely changed regarding information on LTFTT generally (see Table 1).

Trainees reported feeling very slightly less supported by HEE, less valued and more isolated.

Table 1. Survey responses to 5-point scale questions ( 1 = not at all, $5=$ very $)$

$\begin{array}{ll}\text { Spring } 2017 & \text { Autumn } \\ (n=6) \text { mean } & 2017(n=4) \\ \text { score } & \text { mean score }\end{array}$

As a LTFT trainee do you feel well 3.2 supported by HEE Y\&H?

As a LTFT trainee do you fee valued?

As a LTFT trainee do you feel

isolated?

How useful have you found the

HEE Y\&H website for information about LTFT training?

How useful have you found the 2

HEE Y\&H website for information about maternity leave?

How useful have you found the

HEE Y\&H website for information about returning to work?

How useful have you found the 2.5

HEE Y\&H website for information about pastoral support?

HEE = Health Education England; LTFT = less-than-full-time; Y\&H = Yorkshire and the Humber. 


\section{Conclusions}

The results from this study are somewhat counterintuitive. Given the significant improvements and the authors' perception that the information created is easy to navigate, easy to read, covers all major areas and has put CMT within $\mathrm{Y} \& \mathrm{H}$ in a nationally leading position with regards to information dissemination, it's surprising that even the score for trainee-reported usefulness of the website, although improved, is barely above neutral.

There could be a number of potential reasons for the key reported outcomes (feelings of support, value and isolation) not changing and it's likely that the cause is a combination of factors. Possible options include: the WhatsApp group, while providing a forum to share difficulties and seek advice, also shares the burden of issues encountered; WhatsApp groups and websites may not be effective ways to support trainees; increased discussion has allowed trainees to express themselves more freely; scores may be a poor surrogate for actual feelings; low numbers of LTFT trainees in many hospitals can't be overcome by virtual groups; HEE is poorly placed to support LTFT trainees and they may need support on the ground more.

\section{Conflict of interest statement}

None declared. 